\title{
Caractéristiques structurales et écologiques des phytocénoses forestières de la forêt classée d'Itchèdè (Département du Plateau, Sud-est Bénin)
}

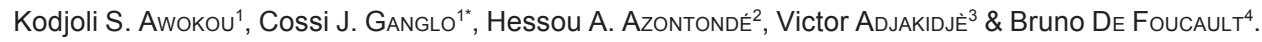 \\ ${ }^{1}$ Université d'Abomey-Calavi, Faculté des Sciences Agronomiques, Département Aménagement et Gestion de l'Environnement. 01 \\ BP 1526 Cotonou, Bénin. \\ 2Institut national des recherches agricoles du Bénin, 01 BP 884 Recette Principale, Cotonou, République du Bénin. \\ ${ }^{3}$ Faculté des Sciences et Techniques. 01 BP 526 Cotonou. République du Bénin. \\ ${ }^{4}$ Département de Botanique, Faculté de Pharmacie. BP 83 F-59006 Lille Cedex. \\ *Auteur pour les correspondances (E-mail : ganglocj@yahoo.fr) \\ Reçu le 03-04-2008, accepté le 24-03-2009.
}

\section{Résumé}

Notre étude phytosociologique a été menée dans la forêt classée d'Itchèdè $\left(6^{\circ} 59^{\prime}-7^{\circ} 00^{\prime} \mathrm{N}\right.$ et $\left.2^{\circ} 37^{\prime}-2^{\circ} 38^{\prime} \mathrm{E}\right)$. Elle a pour objectif de mieux connaître la forêt pour un meilleur suivi de sa dynamique en vue d'asseoir à terme, une base fiable de son aménagement et de sa gestion durable. Nous avons utilisé l'approche synusiale intégrée pour identifier et caractériser les communautés végétales. La topographie et les sols ont été étudiés à travers la mesure des pentes, l'appréciation de la texture tactile à travers des profils texturaux effectués sur $50 \mathrm{~cm}$ de profondeur, la description de profils pédologiques complétée par des analyses d'échantillons de sol au laboratoire. L'étude des paramètres dendrométriques et sylvicoles s'est faite à l'intérieur de placettes circulaires couvrant chacune 0,0314 ha pour les plantations forestières et 0,0615 ha pour la forêt naturelle.

Les résultats ont révélé la présence de six phytocénoses dont quatre phytocénoses non pionnières de sous-bois. Les caractéristiques dendrométriques des phytocénoses permettent de déduire que la forêt naturelle d'Itchèdè est une formation secondaire assez dégradée mais relativement riche en sujets de grands diamètres en comparaison avec les autres forêts du Bénin.

Mots-clés : phytosociologie synusiale, phytocénoses, stations forestières, Itchèdè, Bénin

\section{Abstract}

Structural and ecological characteristics of plant-communities in Itchèdè forest reserve (Plateau Department, South Benin).

The main purpose of the phytosociological study in Itchèdè forest reserve $\left(6^{\circ} 59^{\prime}\right.$ to $7^{\circ} 00^{\prime} N$ and $2^{\circ} 37^{\prime}$ to $\left.2^{\circ} 38^{\prime} \mathrm{E}\right)$ was to contribute to the sustainable management of the forest. Phytosociological survey was done using the synusial approach. Soils and topography were studied through textural profiles appreciation in the first $50 \mathrm{~cm}$ of the soil, pedological profiles description and laboratory analysis. Dendrometric and sivicultural parameters were studied inside circular plots of 0.0314 ha for plantations and 0.0615 ha for natural forest.

The study revealed the existence of six plant-communities including four non pioneer plant-communities were identified and described. Based on dendrometric parameters of the natural forest, we assume that Itchèdè forest reserve is a secondary degraded forest but somewhat rich in trees with high diameters in comparison with other natural forest in Benin.

Key-words : synusial phytosociology, plant-communities, forest sites, Itchèdè, Benin. 


\section{Introduction}

Les ressources forestières du Bénin sont assez limitées. Selon la FAO (2005), les forêts du Bénin couvrent une superficie de 2351000 ha dont 114000 ha de plantations forestières. Ces maigres ressources sont en proie à une dégradation alarmante. En effet, d'après les derniers chiffres de la FAO (2005), le Bénin perd en moyenne 65000 ha de forêts chaque année. Depuis 1940 , ce pays a mis en œuvre une politique de reboisement des aires dégradées pour lutter contre la destruction de ses ressources forestières (Ganglo \& De Foucault, 2006). Les recherches d'accompagnement dont les résultats devraient servir de base aux interventions sylvicoles font défaut. Ainsi, de sérieuses études méritent donc d'être entreprises pour une gestion durable des maigres ressources forestières du Bénin. Par ailleurs, de nombreuses études ont montré que la phytosociologie est une science fiable d'investigation de la végétation car elle permet notamment d'identifier les stations forestières qui sont les unités de base d'un aménagement et d'une gestion durables des forêts (Ganglo, 2005 ; Ganglo \& De Foucault, 2006 ; Noumon \& Ganglo, 2005 ; Noumon et al., 2006 ; Aoudji \& Ganglo, 2006 ; Aoudji et al., 2006 ; Tohngodo et al., 2006 ; 2006). La forêt d'Itchèdè est l'une des rares reliques de forêts naturelles au Sud-Bénin. Un aménagement et une gestion durables s'imposent pour sa sauvegarde et contribuent aussi à la conservation des écosystèmes forestiers et de la biodiversité. Le présent article a pour objectif d'analyser les caractéristiques structurales et écologiques de la forêt d'Itchèdè afin de faire des propositions d'aménagement dans la perspective d'une gestion durable.

\section{Matériel et méthodes}

\section{1-Milieu d'étude}

La forêt classée de Itchèdè $\left(6^{\circ} 59^{\prime}\right.$ et $7^{\circ} 00^{\prime} \mathrm{N}$; $2^{\circ} 37^{\prime}$ et $2^{\circ} 38^{\prime} \mathrm{E}$ ) est située dans la Commune d'Adjaouèrè, dans le Département du Plateau au Sud-Est du Bénin (Fig. 1), avec une superficie estimée à 95 ha. Selon les données de I'ASECNA (Agence pour la Sécurité de la Navigation Aérienne en Afrique et à Madagascar) au Bénin (2006), la forêt est soumise à un climat de type guinéen côtier équatorial ; la pluviométrie moyenne annuelle est $1134 \mathrm{~mm}$ sur une période de 30 ans (1977 - 2006). Sur la même période de 30 ans, les températures moyennes mensuelles varient entre $25^{\circ} \mathrm{C}$ et $29^{\circ} \mathrm{C}$ tandis que l'humidité relative de l'air oscille entre $68 \%$ et $85 \%$. Les sols de cette forêt sont de deux types: les sols ferrallitiques à texture sablo-limoneuse occupant plus de $60 \%$ de la forêt et la terre de barre à texture argilo-sableuse développée dans le continental terminal. La végétation est composée majoritairement de forêt naturelle avec un sous-bois assez diversifié et de plantations de Tectona grandis L.f., de Terminalia superba Engl \& Diels et d'Acacia auriculiformis A. Cunn. ex Benth. Dans la forêt d'Itchèdè, il existe une relation entre la nature de la végétation en place, le type de sol et l'intensité de l'anthropisation du milieu (Awokou, 2004). En effet, sur les sols argilo-sableux soumis à une forte pression anthropique, repose une végétation de forêt dégradée avec un sous-bois arbustivolianescent fait de Olax subscorpioidea Oliv. et de Psydrax horizontalis (K. Schum. \& Thonn.) Bridson. Quand l'action anthropique est de faible intensité, la végétation observée est une formation marquée par la présence de grands arbres comme Ceiba pentandra (L.) Gaert, Triplochiton scleroxylon K. Schum, Antiaris toxicaria Lesch, avec un sousbois composé d'espèces comme Dictyandra arborescens Welw. ex Hook. f. et Rothmannia urcelliformis (Hiern) Bullock ex Robyns. Sur les sols sablo-limoneux où, l'action anthropique a d'ailleurs été presque négligeable, la végétation est une forêt dense avec des ligneux comme Anthonotha macrophylla P., Beauv., Antiaris toxicaria Lesch, Celtis zenkeri Engl., tandis que le sous-bois est dominé par Microdesmis keayana Léonard et Motandra guineense A. DC. Au niveau des plantations, établies majoritairement sur sols sableux, le sous-bois est dominé par Chromolaena odorata (L.) R. King \& $\mathrm{H}$. Robinson et Panicum maximum Jacq. dans les plantations ouvertes et par Mallotus oppositifolius (Geisl.) Müll. Arg. et Rytigynia canthioides (Benth.) Robyns dans les plantations fermées. 


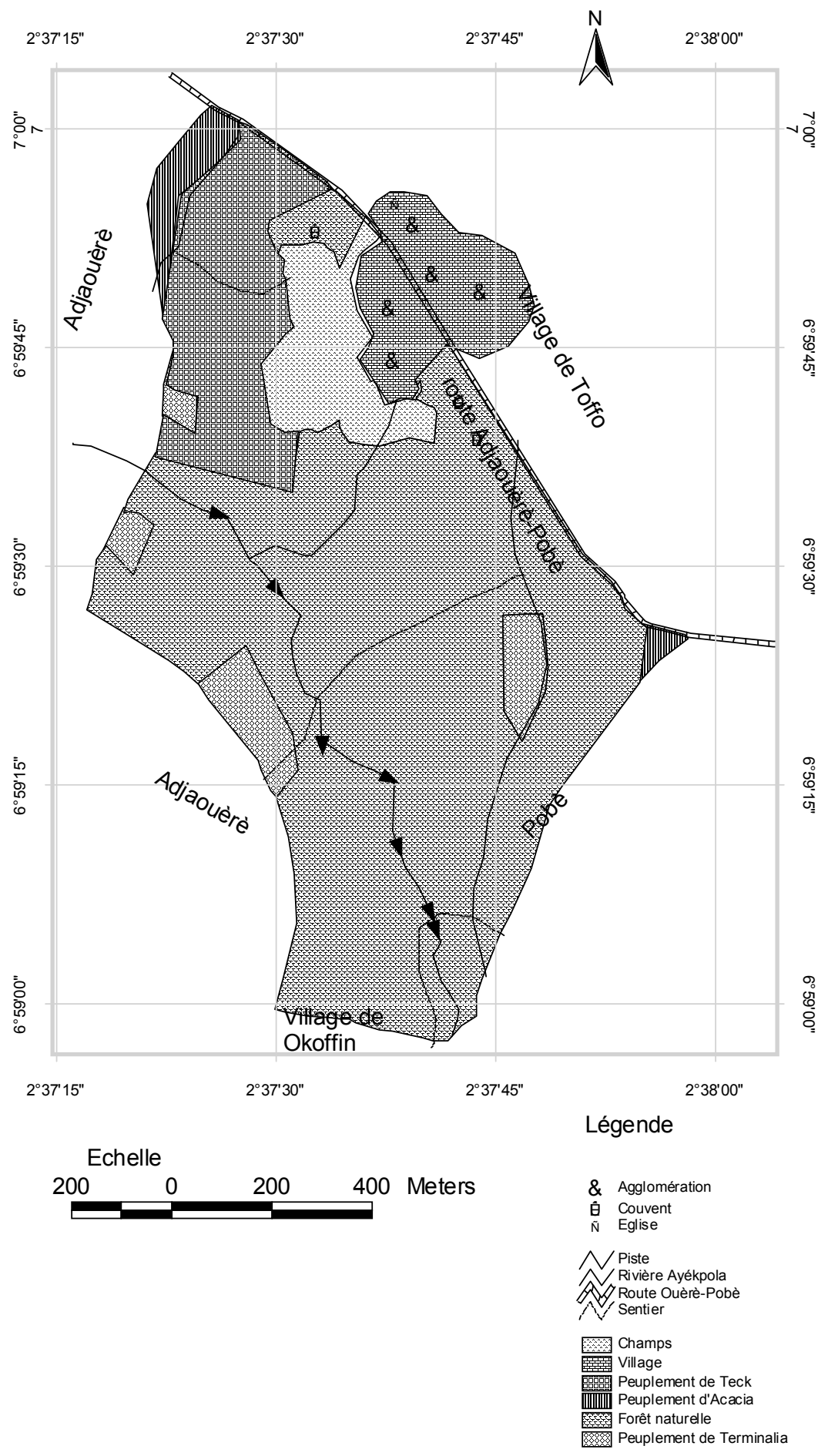

Figure 1 : Vue globale de la forêt d'Itchèdè 


\subsection{Méthodologie}

Pour effectuer l'étude phytosociologique de la végétation, nous avons utilisé une carte de végétation de la forêt pour faciliter notre orientation au sein de la forêt. Le "Global Positioning System" (GPS) nous a permis de matérialiser divers points repères et de cartographier les phytocénoses identifiées.

Les mesures de diamètres et hauteurs d'arbres ont été effectuées grâce au mètre ruban et au clinomètre SUUNTO. Pour étudier le sol et la topographie, nous avons eu recours à la tarière pédologique et au clinomètre SUUNTO (mesure de pente)

L'étude phytosociologique de la végétation spontanée a été réalisée suivant l'approche de la phytosociologie synusiale intégrée développée par Gillet et al. (1991) et Gillet (2000). Cette approche comprend deux étapes. La première étape synusiologique vise à décrire, classer et comprendre le déterminisme des synusies végétales. Ainsi, la répétition des combinaisons d'espèces, en relation avec les facteurs du milieu (sol, position topographique, pente, ....), permet d'identifier les synusies végétales. La deuxième étape, phytocénologique, permet de définir les phytocénoses qui sont des combinaisons de synusies interdépendantes. Les aires minimales des relevés sont de $500 \mathrm{~m}^{2}$ pour les synusies annuelles et herbacées vivaces et de $1000 \mathrm{~m}^{2}$ pour les synusies arbustives et arborescentes. Pour chaque relevé phytosociologique, les spermaphytes présentes et leur coefficient d'abondance dominance sont notés. Sur la base des relations spatio-temporelles qui existent entre les synusies végétales, elles ont été intégrées en phytocénoses.

L'étude des paramètres dendrométriques a été réalisée à l'optimum des phytocénoses à l'intérieur de placettes circulaires de $314 \mathrm{~m}^{2}$ en plantation forestière et $615 \mathrm{~m}^{2}$ en forêt naturelle, l'objectif étant d'avoir au moins 10 à 12 arbres par placette comme recommandé par Duplat et Perrote (1981). Au sein de chaque placette, dans les plantations forestières, nous avons mesuré, la circonférence à hauteur d'homme $(1,3 \mathrm{~m}$ de hauteur à partir $\mathrm{du}$ niveau du sol) de tous les arbres, la hauteur des deux plus gros arbres de la placette et celle de deux arbres situés dans la classe moyenne de diamètre. Dans les forêts naturelles, nous avons mesuré, la circonférence à hauteur d'homme $(1,3$ $\mathrm{m}$ de hauteur à partir du niveau du sol) des arbres ayant au moins $10 \mathrm{~cm}$ de circonférence à hauteur d'homme. Les caractéristiques dendrométriques des peuplements ont été calculées.

L'étude du sol a été faite par la description de profil textural réalisé sur les 50 premiers centimètres de profondeur, complété par des profils pédologiques de $2 \mathrm{~m}$ de long, $1 \mathrm{~m}$ de large et $2 \mathrm{~m}$ de profondeur dont la description a été complétée par des analyses de laboratoire (Ganglo \& De Foucault, 2006).

\section{Résultats}

\section{1-Phytocénoses identifiées}

Sur la base des relations spatio-temporelles entre les synusies identifiées, celles-ci ont été intégrées en six phytocénoses:

Phytocénose à Chromolaena odorata (L.) R. King \& H. Robinson et Panicum maximum Jacq. ; elle se développe sur les sols ferralitiques sablo-limoneux ou latéritiques ; c'est une phytocénose pionnière héliophile à conditions stationnelles variables.

- Phytocénose à Bambusa vulgaris Schrad ex., Wendel ; elle se rencontre sur les sols ferrallitiques sablo-limoneux ou argilo-sableux.

Phytocénose à Mallotus oppositifolius (Geisl.) Müll. Arg. et Rytigynia canthioides (Benth.) Robyns; elle connaît son optimum de développement sur les sols ferrallitiques sablo-limoneux.

- Phytocénose à Olax subscorpioidea Oliv. et Psydrax horizontalis (K. Schum. \& Thonn.) Bridson; elle se développe sur les sols ferrallitiques argilo-sableux.

- Phytocénose à Rothmannia urcelliformis (Hiern) Bullock ex Robyns et Dictyandra arborescens Welw. ex Hook. f. ; on la rencontre sur les sols ferrallitiques argilo-sableux.

Phytocénose à Anthonotha macrophylla P. Beauv. et Antiaris toxicaria Lesch. ; elle caractérise les sols ferrallitiques sablolimoneux. 
Les caractéristiques stationnelles et structurales des phytocénoses sont présentées au tableau 1. D'après ce tableau, sur l'ensemble structural composé des sujets ayant au moins $10 \mathrm{~cm}$ de diamètre à hauteur d'homme, les densités des phytocénoses varient entre 146 et 338 pieds/ha pour une surface terrière de 13 à $43 \mathrm{~m}^{2} /$ ha (tableau 1). Les phytocénoses sont représentées à la figure 2.

Tableau 1 : Synthèse des principales caractéristiques structurales et écologiques des phytocénoses de la forêt d'Itchèdè.

\begin{tabular}{|c|c|c|}
\hline Phytocénoses & Conditions stationnelles & Caractéristiques structurales \\
\hline $\begin{array}{l}\text { Phytocénose à } \\
\text { Chromolaena odorata } \\
\text { (L.) R. King. \& H. } \\
\text { Robinson et Panicum } \\
\text { maximum Jacq. }\end{array}$ & $\begin{array}{l}\text { Sols ferralitiques sablo-limoneux ou } \\
\text { latéritiques; phytocénose pionnière } \\
\text { héliophile à conditions stationnelles } \\
\text { variables. }\end{array}$ & $\begin{array}{l}\text { Phytocénose retrouvée sous une } \\
\text { plantation de Terminalia superba Engl. } \\
\& \text { Diels de } 11 \text { ans d'âge avec }: \mathrm{N}= \\
518 \text { pieds } / \mathrm{ha} ; \mathrm{Hg}=17,19 \mathrm{~m} ; \mathrm{Hd}=20 \\
\mathrm{~m} ; \mathrm{Dg}=18,63 \mathrm{~cm} ; \mathrm{S}=21,69 \% \text { et } \mathrm{G} \\
=16,5 \mathrm{~m}^{2} / \mathrm{ha}\end{array}$ \\
\hline $\begin{array}{l}\text { Phytocénose à } \\
\text { Bambusa vulgaris } \\
\text { Schrad. ex Wendel }\end{array}$ & $\begin{array}{l}\text { Sols ferrallitiques sablo-limoneux ou } \\
\text { argilo-sableux ; taux de matière } \\
\text { organique }=1,84 \text { à } 4,82 \% \text {; rapport } \\
\mathrm{C} / \mathrm{N}=16,3 \text { à } 38,3 ; \mathrm{pH}=5,17 \text { à } \\
6,33 ; \mathrm{CEC}=11 \text { à } 17 \text { méq } / 100 \mathrm{~g} .\end{array}$ & Phytocénose pionnière non plantée \\
\hline $\begin{array}{l}\text { Phytocénose à Mallotus } \\
\text { oppositifolius (Geisl.) } \\
\text { Müll. Arg. et Rytigynia } \\
\text { canthioides (Benth.) } \\
\text { Robyns }\end{array}$ & $\begin{array}{l}\text { sol ferrallitique sablo-limoneux } \\
\text { taux de matière organique }=1,22 \text { à } \\
1,89 \% ; \text { rapport } \mathrm{C} / \mathrm{N}=12,1 \text { à } 16,9 ; \\
\mathrm{pH}=5,7 \text { à } 6,58 ; \mathrm{CEC}=12 \text { à } 16 \\
\text { méq } / 100 \mathrm{~g} .\end{array}$ & $\begin{array}{l}\text { Phytocénose retrouvée sous une } \\
\text { plantation de Tectona grandis } \mathrm{L} \text {. f. de } \\
39 \text { ans d'âge avec }: \mathrm{N}=381 \text { pieds/ha } \\
\mathrm{Hg}=25,39 \mathrm{~m} ; \mathrm{Hd}=29 \mathrm{~m} ; \mathrm{Dg}=31,38 \\
\mathrm{~cm} ; \mathrm{S}=17,68 \% \text { et } \mathrm{G}=29,72 \mathrm{~m}^{2} / \mathrm{ha}\end{array}$ \\
\hline $\begin{array}{l}\text { Phytocénose à Olax } \\
\text { subscorpioidea Oliv. et } \\
\text { Psydrax horizontalis (K. } \\
\text { Schum. \& Thonn.) } \\
\text { Bridson }\end{array}$ & $\begin{array}{l}\text { Sol ferrallitique argilo-sableux ; taux } \\
\text { de matière organique }=2,18 \text { à } 6 \% ; \\
\text { rapport } \mathrm{C} / \mathrm{N}=16,3 \text { à } 20,8 ; \mathrm{pH}= \\
5,79 \text { à } 5,94 ; \mathrm{CEC}=12 \text { à } 15 \\
\text { méq } / 100 \mathrm{~g}\end{array}$ & $\begin{array}{l}\text { Phytocénose retrouvée sous forêt } \\
\text { naturelle avec } N=146 \text { pieds } / \text { ha }(\mathrm{dbh} \geq \\
10 \mathrm{~cm}) ; \mathrm{G}=13,85 \mathrm{~m}^{2} / \mathrm{ha} ; \mathrm{Dg}=34,13 \\
\mathrm{~cm} .\end{array}$ \\
\hline $\begin{array}{l}\text { Phytocénose à } \\
\text { Rothmannia } \\
\text { urcelliformis (Hiern) } \\
\text { Bullock ex Robyns et } \\
\text { Dictyandra } \\
\text { arborescens Welw. ex } \\
\text { Hook. f. }\end{array}$ & $\begin{array}{l}\text { Sol ferrallitique argilo-sableux ; taux } \\
\text { de matière organique }=4,47 \text { à } 4, \\
59 \% ; \text { rapport } \mathrm{C} / \mathrm{N}=17,3 \text { à } 44,1 ; \mathrm{pH} \\
=5,05 \text { à } 6,2 ; \mathrm{CEC}=13 \text { à } 26 \\
\text { méq } / 100 \mathrm{~g}\end{array}$ & $\begin{array}{l}\text { Phytocénose retrouvée sous forêt } \\
\text { naturelle avec } N=277 \text { pieds } / \text { ha }(\mathrm{dbh} \geq \\
10 \mathrm{~cm}) ; \mathrm{G}=30,72 \mathrm{~m}^{2} / \mathrm{ha} ; \mathrm{Dg}=41,20 \\
\mathrm{~cm} .\end{array}$ \\
\hline $\begin{array}{l}\text { Phytocénose à } \\
\text { Anthonotha macrophylla } \\
\text { P. Beauv. et Antiaris } \\
\text { toxicaria Lesch. }\end{array}$ & $\begin{array}{l}\text { Sol ferrallitique sablo-limoneux ; taux } \\
\text { de matière organique }=1,01 \text { à } \\
4,64 \% \text {; rapport } \mathrm{C} / \mathrm{N}=16,5 \text { à } 22,9 ; \\
\mathrm{pH}=5,81 \text { à } 6,16 ; \\
\mathrm{CEC}=7 \text { à } 13 \text { méq } / 100 \mathrm{~g} .\end{array}$ & $\begin{array}{l}\text { Phytocénose retrouvée sous forêt } \\
\text { naturelle avec } N=338 \text { pieds } / \text { ha }(\mathrm{dbh} \geq \\
10 \mathrm{~cm}) ; \mathrm{G}=42,97 \mathrm{~m}^{2} / \mathrm{ha} ; \mathrm{Dg}=38 \mathrm{~cm} \text {. }\end{array}$ \\
\hline
\end{tabular}

$\underline{N B}: N=$ densité du peuplement arborescent en nombre de pieds $/$ ha $; \mathrm{Dg}=$ diamètre moyen du peuplement arborescent $; \mathrm{Hg}=$ hauteur moyenne ; $G$ = surface terrière du peuplement arborescent en $\mathrm{m}^{2} / \mathrm{ha} ; \mathrm{S}=$ facteur d'espacement exprimé en \%. 


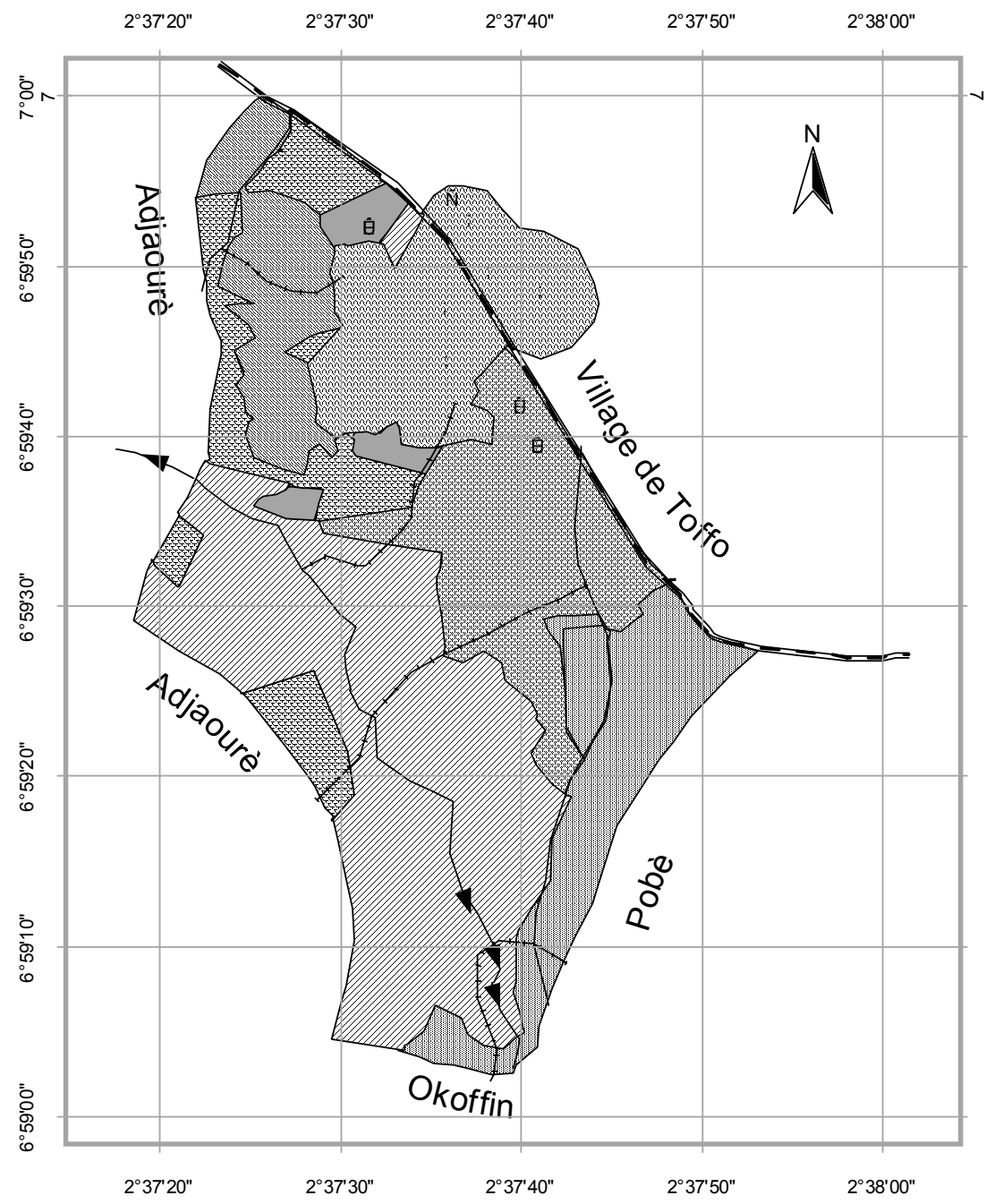

Echelle

\section{$100 \quad 0 \quad 100200$ Meters}

Légende

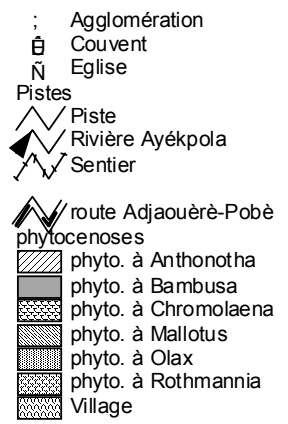

Figure 2 : Phytocénoses de la forêt d'Itchèdè 
Les figures 3,5 et 7 présentent les structures diamétriques des peuplements arborescents de trois phytocénoses forestières de la forêt d'Itchèdè. Ces figures montrent une allure exponentielle décroissante classique de la structure diamétrique de ces peuplements forestiers. La structure diamétrique est cependant plus régulière dans la phytocénose à Anthonotha macrophylla $\mathrm{P}$. Beauv. et Antiaris toxicaria Lesch (Fig. 7) que dans les deux autres phytocénoses (Fig. 3 et 5). C'est également dans cette phytocénose qu'on note le meilleur ajustement, de la structure diamétrique, à la fonction exponentielle avec le coefficient de détermination le plus élevé $\left(R^{2}=0,97\right)$. Dans la phytocénose à Olax subscorpioidea Oliv. et Psydrax horizontalis (K. Schum. \& Thonn.) Bridson (Fig. 3), on note une faible densité dans la première classe de diamètre; ceci traduit un problème de régénération des espèces de cette phytocénose. Dans la phytocénose à Rothmannia urcelliformis (Hiern) Bullock ex Robyns et Dictyandra arborescens Welw. ex
Hook. f. (Fig. 5), on note un déficit de densité dans les dernières classes de diamètre. Ceci peut être dû à l'impact d'une exploitation frauduleuse des gros arbres du peuplement.

Les figures 4, 6 et 8 , sont une pondération des figures 3, 5 et 7 par la surface terrière. A cet effet, les gros sujets des dernières classes de diamètre pèsent d'un poids important dans la structure diamétrique en fonction de la surface terrière. Les meilleurs ajustements à la structure diamétrique en fonction de la surface terrière sont obtenus avec des fonctions polynomiales d'ordre 3. Le coefficient de détermination le plus élevé $(0,9707)$ est obtenu dans la phytocénose à Olax subscorpioidea Oliv. et Psydrax horizontalis (K. Schum. \& Thonn.) Bridson (Fig. 4).

Selon la répartition des surfaces terrières en fonction des classes de diamètre dans les phytocénoses forestières d'Itchèdè (Fig. 4, 6 et 8 ) les peak des surfaces terrières sont obtenus entre 35 et $55 \mathrm{~cm}$.

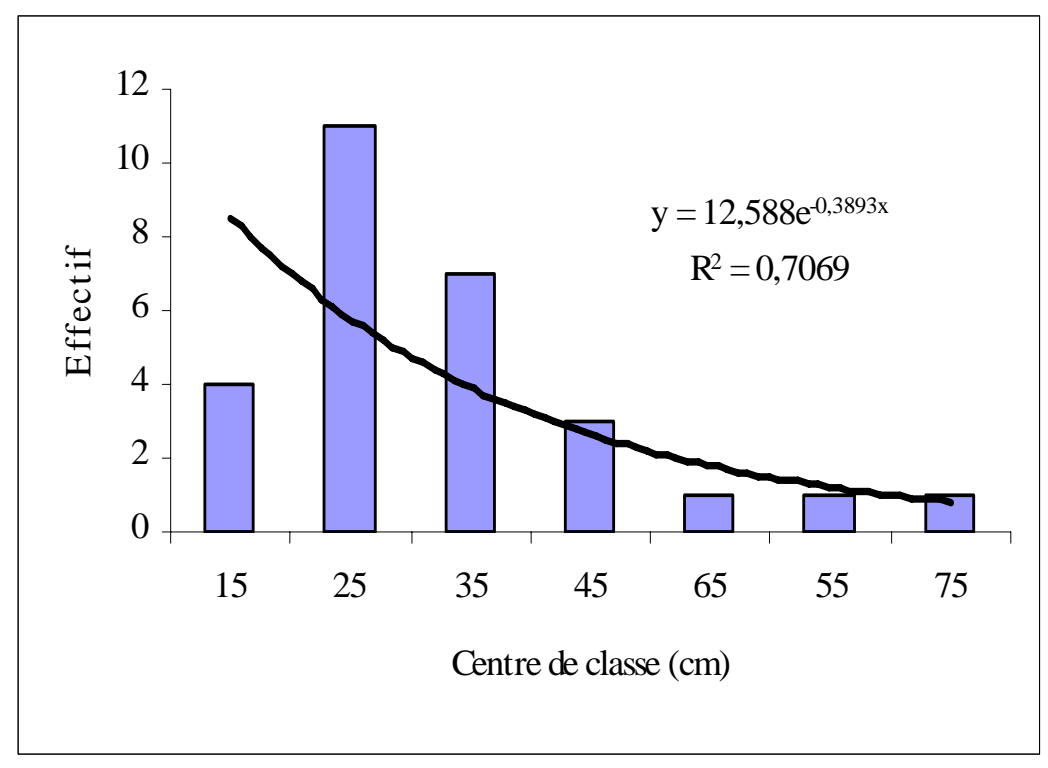

Figure 3 : Répartition par classe de diamètre du peuplement arborescent de la phytocénose à Olax subscorpioidea et Psydrax horizontale 


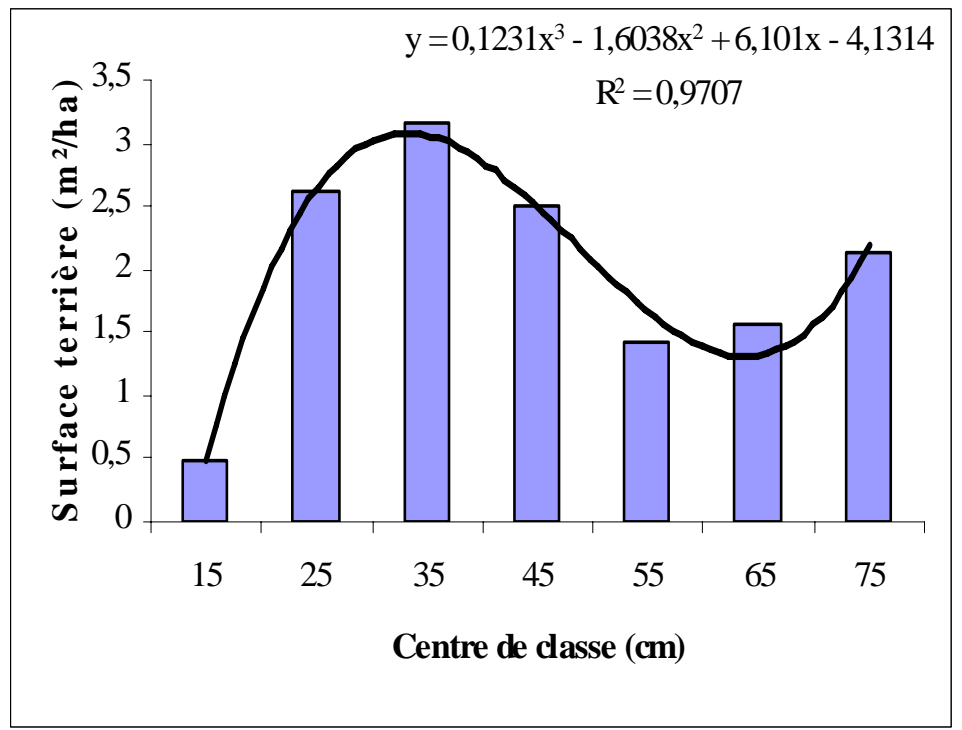

Figure 4 : Répartition par classe de diamètre de la surface terrière du peuplement arborescent de la phytocénose à Olax subscorpioidea et Psydrax horizontale

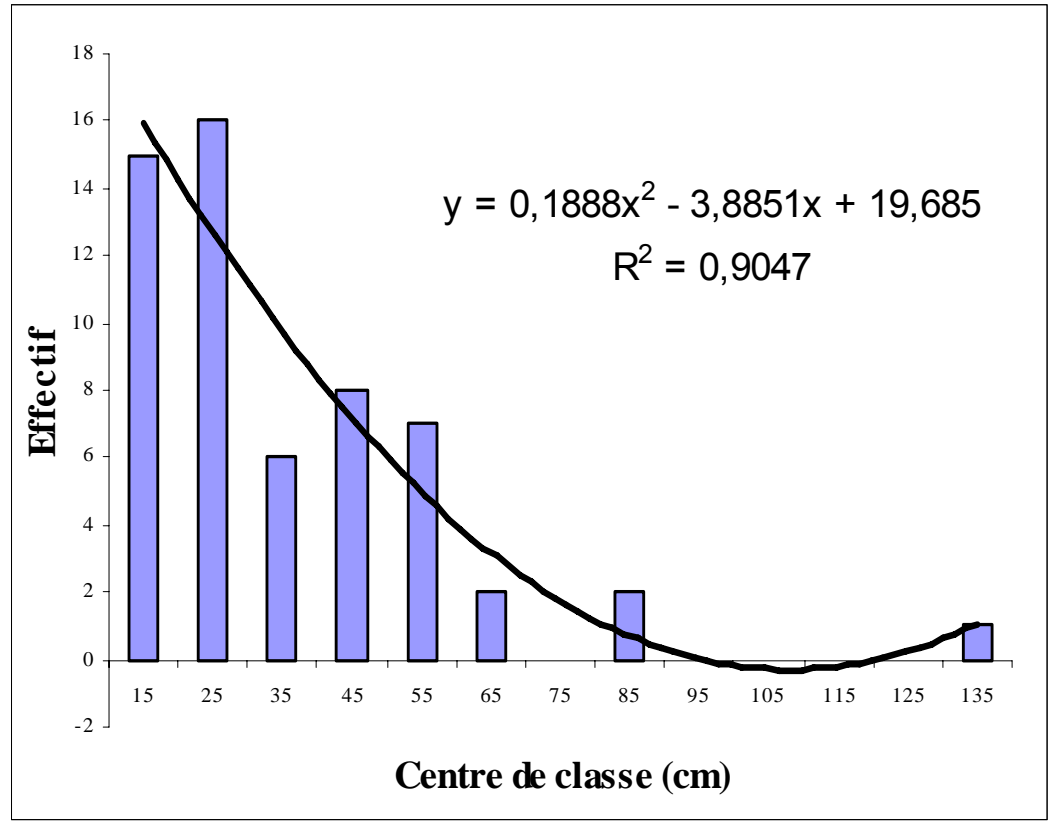

Figure 5 : Répartition par classe de diamètre du peuplement arborescent de la phytocénose à Rothmannia urcelliformis et Dictyandra arborescens 


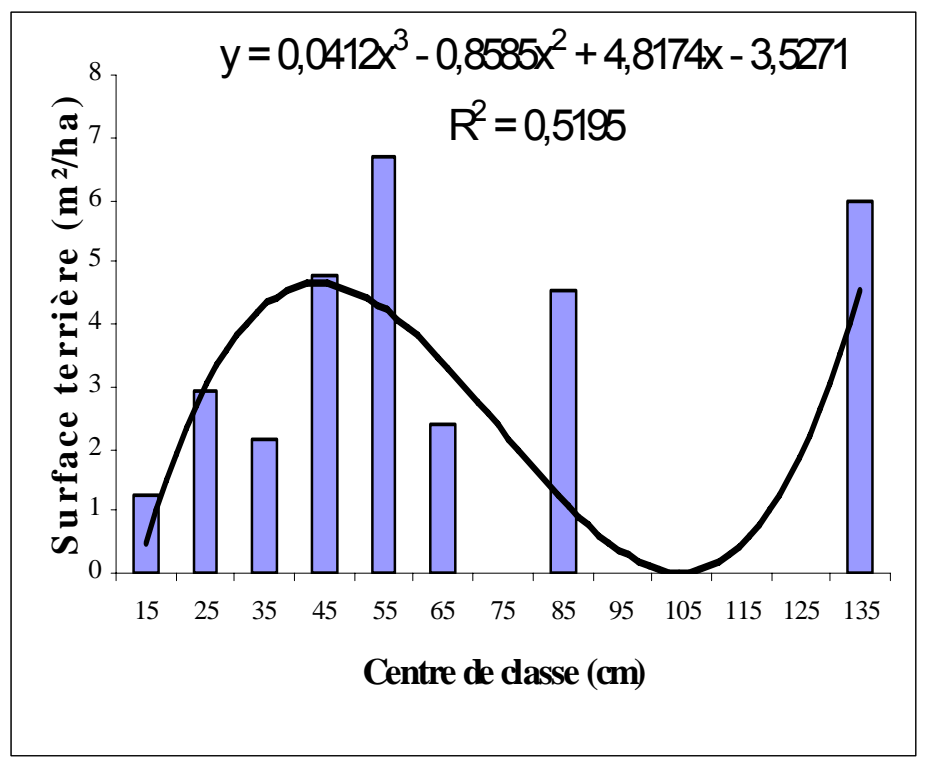

Figure 6 : Répartition par classe de diamètre de la surface terrière du peuplement arborescent de la phytocénose à Rothmannia urcelliformis et Dictyandra arborescens

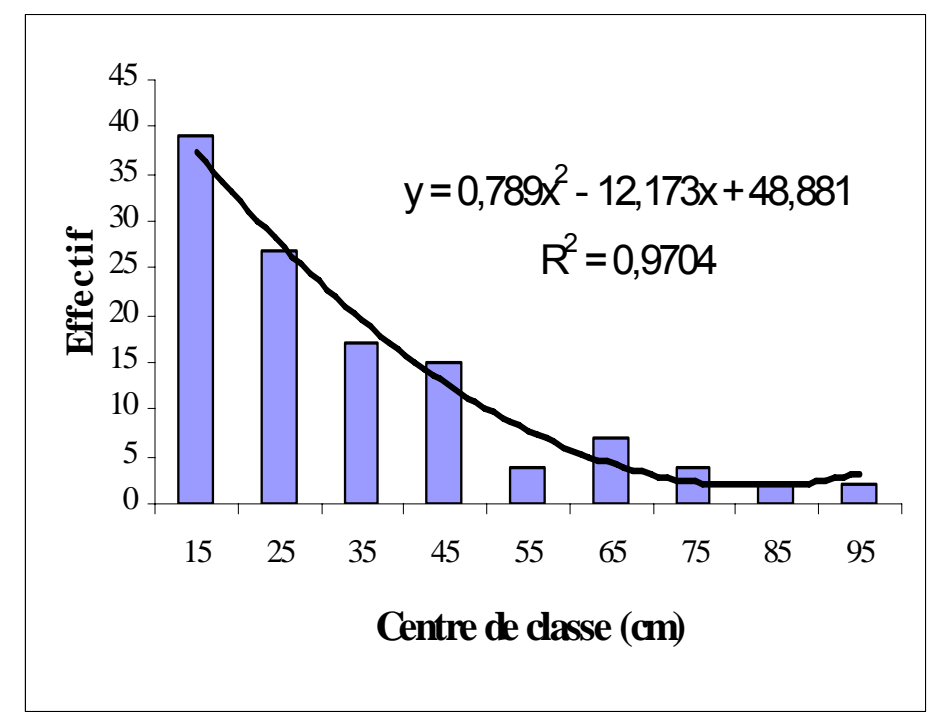

Figure 7 : Répartition par classe de diamètre du peuplement arborescent de la phytocénose à Anthonotha macrophylla et Antiaris toxicaria 


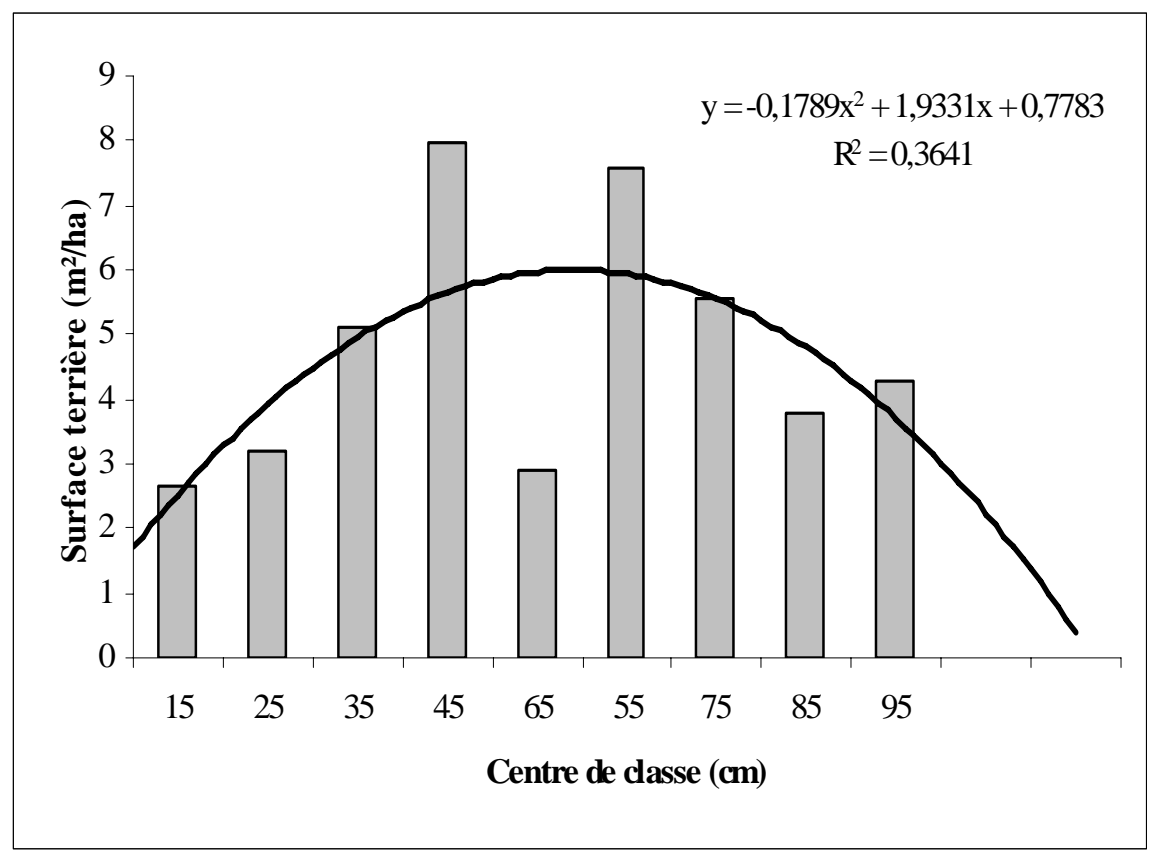

Figure 8 : Répartition par classe de diamètre de la surface terrière du peuplement arborescent de la phytocénose à Anthonotha macrophylla et Antiaris toxicaria

\subsection{Stations forestières identifiées}

Les biotopes des phytocénoses non pionnières de sous-bois identifiées dans la forêt d'Itchèdè sont caractérisés par une homogénéité remarquable des conditions stationnelles et des caractéristiques structurales.

Ces constats nous amènent à considérer les biotopes des phytocénoses non pionnières de sous-bois de la forêt d'Itchèdè comme des stations forestières.

Plusieurs stations forestières ont été ainsi identifiées :

- la station représentée par le biotope de la synusie arbustive à Microdesmis keayana Léonard et Motandra guineensis (Thonning) A. DC. dans la forêt à
Anthonotha macrophylla P. Beauv. et Antiaris toxicaria Lesch ;

- la station représentée par le biotope de la phytocénose à Rothmannia urcelliformis (Hiern) Bullock ex Robyns et Dictyandra arborescens Welw. ex Hook. f. ;

- la station représentée par le biotope de la Phytocénose à Olax subscorpioidea Oliv. et Psydrax horizontalis (K. Schum. \& Thonn.) Bridson ;

- la station représentée par le biotope de la phytocénose à Mallotus oppositifolius (Geisl.) Müll. Arg. et Rytigynia canthioides (Benth.) Robyns.

Les caractéristiques écologiques de ces stations sont présentées au tableau 1. 


\section{4. discussion}

\subsection{Déterminisme écologique des phyto- cénoses}

La phytocénose est une communauté végétale formée d'un ensemble de synusies organisées spatialement, temporellement et fonctionnellement (Gillet et al., 1991). Ces synusies sont liées par des relations de dépendance d'ordre écologique, dynamique et génétique. Ainsi, les facteurs écologiques que sont la topographie et le sol ont joué un rôle capital dans le déterminisme des diverses phytocénoses. Le développement de façon prioritaire de la phytocénose à Olax subscorpioidea Oliv. et Psydrax horizontalis (K. Schum. \& Thonn.) Bridson sur plateau illustre l'influence de la topographie dans la mise en place des phytocénoses. De la même manière, la phytocénose à Rothmannia urcelliformis (Hiern) Bullock ex Robyns et Dictyandra arborescens Welw. ex Hook. f. qui est restée inféodée au sol argileux ou argilo-sableux, nous amène à dire que les conditions édaphiques influent fortement sur la mise en place des phytocénoses. La richesse en eau du sol est également un élément déterminant dans la mise en place des phytocénoses. Ainsi, sur les sols hydromorphes sablo-limoneux, situés en bordure de la rivière Ayékpola, il se développe une végétation luxuriante assez diversifiée avec une grande représentation des ligneux de grande taille. Anthonotha macrophylla P. Beauv., qui est l'espèce dominante de la phytocénose qui s'y développe, n'est jamais retrouvée sur le plateau. Cette phytocénose est très proche de la forêt à Cleistopholis patens (Benth.) Engl. \& Diels et Ficus mucuso Filcalho décrite par Sokpon (1995) qui signalait déjà que ce groupement ne se retrouve que dans les basfonds et à mi-pente sur sols hydromorphes à texture sableuse mais, jamais au sommet du plateau sur sol argileux. Notre étude nous amène à conclure que la topographie et les types de sols sont des facteurs écologiques qui jouent un rôle capital dans la mise en place des communautés végétales. Notre conclusion corroborent ainsi les résultats des travaux antérieurs dans le domaine de l'étude des communautés végétales (Sokpon, 1995 ; Ganglo et al., 1999 ; Ganglo \& De Foucault, 2006 ; Ganglo, 2005 ; Aoudji \& Ganglo, 2006 ; Aoudji et al., 2006 et Noumon \& Ganglo, 2005 ; Noumon et al., 2006).

\subsection{Caractéristiques structurales des phytocénoses}

La structure de la forêt d'Itchèdè révèle une faible densité tant en nombre d'arbres qu'en surface terrière à l'hectare. Cette forêt paraît ainsi plus dégradée que celle de Pobè située à la même latitude où, pour les arbres ayant au moins $5 \mathrm{~cm}$ de diamètre à hauteur d'homme, Sokpon (1995) a obtenu une densité variant entre 648 et 1014 pieds ha-1 $^{-1}$ pour une surface terrière de 40 à $44 \mathrm{~m}^{2} \mathrm{ha}^{-1}$.

La forêt d'Itchèdè a cependant une surface terrière comparable à celle des formations forestières et savanicoles de l'Ouémé-Boukou au centre du Bénin où, Hessou (2003), sur des sujets ayant au moins $10 \mathrm{~cm}$ de diamètre à hauteur d'homme, a enregistré des densités de 276 à 589 pieds/ha pour des surfaces terrières variant entre 9 et $31 \mathrm{~m}^{2} \mathrm{ha}^{-1}$.

Dans la forêt des Monts-Kouffé (nord-est du Bénin), Hunhyet (2000) a obtenu une densité assez proche de celle de la forêt d'Itchèdè mais, la surface terrière y est plus faible; en effet, d'après cet auteur, la densité de la forêt des Monts-Kouffé varie entre 104 et 328 pieds ha-1 ${ }^{-1}$ pour une surface terrière de 3 à $25 \mathrm{~m}^{2}$ sur les sujets ayant plus de $10 \mathrm{~cm}$ de diamètre à hauteur d'homme.

Les forêts denses sèches à Celtis $s p$ et à Anogeissus leiocarpa (DC) Guill. \& Perr. étudiées en Côte d'Ivoire par Devineau (1984) sont également plus denses que celle d'Itchèdè ; en effet, les densités de ces forêts sont comprises entre 1236 et 2884 pieds ha ${ }^{-1}$ pour des surfaces terrières variant entre 34 et $38 \mathrm{~m}^{2} \mathrm{ha}^{-1}$ pour des sujets ayant au moins 5 $\mathrm{cm}$ de diamètre à hauteur d'homme.

Selon Mosango (1990) et Sokpon (1995), le peak de la répartition de la surface terrière par classe de diamètre est un critère de choix pour juger du stade de l'évolution et de l'exploitation des forêts. Ainsi, le peak de la courbe croît en fonction de l'âge ou du stade d'évolution du peuplement $(35 \mathrm{~cm}$ pour la forêt secondaire jeune ; $65 \mathrm{~cm}$ pour la forêt secondaire vieille et plus de $65 \mathrm{~cm}$ pour la forêt primaire). Les peak des surfaces terrières des phytocénoses de la forêt d'Itchèdè étant obtenus entre 35 et 
$55 \mathrm{~cm}$, nous en déduisons que ces phytocénoses forestières évoluent du stade de jeune forêt secondaire vers celui de vieille forêt secondaire.

Du point de vue du diamètre moyen, les phytocénoses forestières d'Itchèdè ont entre 34 et $41 \mathrm{~cm}$. Ces valeurs sont assez comparables à celles de la forêt de Pobè $(29$ à $38 \mathrm{~cm}$ ) (Sokpon, 1995). Elles sont par contre plus élevées que celles mesurées dans d'autres forêts du Bénin. En effet, dans les forêts de l'Ouémé-Boukou au centre du Bénin, les diamètres moyens varient entre 20 et $27 \mathrm{~cm}$ pour les sujets ayant plus de $10 \mathrm{~cm}$ de diamètre à hauteur d'homme, tandis que dans la forêt des Monts-Kouffé au nord-ouest du Bénin, Hunhyet (2000) a obtenu sur le même ensemble structural, entre 18 et $33 \mathrm{~cm}$ de diamètre moyen. Dans la forêt de Bassila au nord-ouest du Bénin, Biaou (1999) a obtenu entre 19 et $30 \mathrm{~cm}$ sur les arbres de plus de $10 \mathrm{~cm}$ de diamètre à hauteur d'homme.

Les caractéristiques dendrométriques ci-dessus énumérées, permettent d'affirmer que la forêt naturelle d'Itchèdè est une formation secondaire assez dégradée mais relativement riche en sujets de grands diamètres en comparaison avec les autres forêts du Bénin.

La structure diamétrique des phytocénoses forestières d'Itchèdè (Fig. 3,5 et 7 ) a en général une allure exponentielle décroissante et paraît ainsi conforme à celle des forêts étudiées par de nombreux auteurs (Devineau, 1984 ; Sokpon, 1995 ; Biaou, 1999; Hunhyet, 2000 ; Hessou, 2003...).

Les caractéristiques dendrométriques de la plantation à Terminamlia superba Engl. \& Diels qu'on retrouve dans la phytocénose à Chromolaena odorata (L.) R. King. \& R. Robinson (tableau 1) montrent que l'accroissement moyen annuel en diamètre est de 1,69 $\mathrm{cm}$ tandis que celui de la hauteur moyenne est de $1,56 \mathrm{~cm}$. Cette plantation fait ainsi partie des plus performantes, de l'Afrique de l'Ouest et de l'Afrique Centrale (Groulez \& Wood, 1984). La plantation de teck, avec une hauteur dominante de $29 \mathrm{~m}$ à l'âge de 39 ans, fait partie de la deuxième classe de productivité définie pour les teckeraies du sud et du centre Bénin (Ganglo et al., 1999) et de la troisième classe de productivité des plantations de teck de la Côted'Ivoire (Dupuy et al., 1999).

\subsection{Proposition d'aménagement de la forêt d'Itchèdè}

Les mesures d'aménagement que nous proposons sont spécifiques à chaque phytocénose non pionnière de sous-bois dont le biotope constitue en soi une station forestière.

La station de la forêt à Mallotus oppositifolius (Geisl.) Müll. Arg. Rytigynia canthioides (Benth.) Robyns repose sur un sol ferrallitique assez profond bénéficiant d'une alimentation hydrique très importante $\mathrm{du}$ fait de la proximité de la nappe phréatique. Le sol a une bonne richesse en élément chimique et un $\mathrm{pH}$ proche de la neutralité $(5,7$ à 6,58$)$. II s'agit également d'un sol non exposé à l'érosion car il est situé sur des pentes faibles. La station forestière à Mallotus oppositifolius (Geisl.) Müll. Arg. et Rytigynia canthioides (Benth.) Robyns est prédisposée à une production de bois d'œurre de grande qualité. Nous recommandons par conséquent des interventions sylvicoles pour aider au développement des semis des espèces naturelles qui s'y retrouvent.

Les sols de la forêt à Olax subscorpioidea Oliv. et Psydrax horizontalis (K. Schum. \& Thonn.) Bridson ont une bonne capacité de rétention en eau. Le $\mathrm{pH}$ est légèrement acide $(5,79$ à 5,94$)$ et la richesse azotée du sol est assez bonne $(0,78$ à $1,68 \%)$. La station présente cependant des terrains pentus (pentes modérées) exposés à l'érosion pluviale. La minéralisation de la matière organique est faible (rapport $\mathrm{C} / \mathrm{N}$ élevé 16,3 à 20,8). Une seule essence locale noble tente de s'y régénérer. II s'agit de Afzelia africana Sm. La production du bois d'œuvre, notamment à partir du Terminalia superba Engl. \& Diels, espèce de pleine lumière ayant donné de bons résultats dans les forêts denses semidécidues après coupe rase est indiquée sur ce terrain. Mais avant, il faut améliorer la structure du sol et limiter les risques d'érosion pluviale par la mise en place initiale d'une plantation de Acacia auriculiformis A. Cunn. ex Benth. par exemple qui produira non seulement une abondante litière mais limitera également l'érosion grâce à son système d'enracinement traçant. 
La station de la forêt à Rothmannia urcelliformis (Hiern) Bullock ex Robyns et Dictyandra arborescens Welw. ex Hook. f. est caractérisée par un sol assez profond et très riche en éléments chimiques. Son sous-bois est marqué par une brosse de régénération d'espèces locales que sont: Antiaris toxicaria Lesh., Triplochiton scleroxylon K. Schum., Ceiba pentandra (L.) Gaert., Albizia glaber-rima (Schum. \& Thonn.) Benth., Millettia thonningii (Shum \& Thonn.) Bak. et Dialium guineense Willd.. Sa strate arborescente est constituée d'essences de valeur telles que Triplochiton scleroxylon K. Shum., Antiaris toxicaria Lesch., Albizia spp et notamment de gros sujets de Ceiba pentandra (L.) Gaert. II s'agit donc d'une station qui mérite d'être conservée. Les opérations sylvicoles consisteront en un enrichissement de la station tout en favorisant l'émergence des espèces locales présentes par des opérations de dépressage et de délianage.

La station à Microdesmis keayana Léonard et Motandra guineensis (Thonning) A. DC. est hydromorphe et offre aux plantes une disponibilité permanente en eau facilement utilisable. Sa position topographique, quasiment en bas de pente lui procure une richesse en matière organique assez importante. C'est la station la plus diversifiée qui regorge de nombreux arbres. Son emplacement au cœur de la forêt, assez éloigné des habitations fait qu'elle est moins soumise à la pression humaine. Cette station peut être érigée en réserve de conservation de la biodiversité de la forêt.

\section{Conclusion}

L'étude phytosociologique de la forêt d'Itchèdè a permis d'identifier et de caractériser six phytocénoses dont deux pionnières. L'étude des facteurs écologiques a montré une homogénéité des conditions stationnelles au sein de chaque phytocénose non pionnière de sous-bois. Les biotopes des phytocénoses non pionnières de sous-bois sont assimilées à des stations forestières. A chacune des stations forestières ainsi identifiées, nous avons formulé des mesures d'aménagement qui tiennent compte de leurs atouts et contraintes. La phytosociologie a donc été pour nous, un outil efficace d'investigation de la végétation.

\section{Remerciements}

C'est un insigne honneur pour nous de remercier les Responsables de l'Ambassade de France et de ceux de l'Institut de Recherche pour le Développement (IRD-France) pour leur soutien financier à nos travaux de recherche à travers le Projet de Coopération pour la Recherche Universitaire et Scientifique (CORUS).

\section{Références citées}

Aoudji A.K.N., \& Ganglo C.J. 2006. Phytosociologie appliquée à l'aménagement des forêts : cas du périmètre forestier de Pahou (Département de l'Atlantique, Sud-Bénin). Journal de Botanique de la Société Botanique de France. 34 : 89-92.

Aoudji A.K.N., Ganglo C.J., Adjakidjè V., de Foucault B. \& Azontondé A.H., 2006. Phytocénose à Barteria nigritana Hook.f. et Rauvolfia vomitoria Afzel. dans le sous-bois des plantations de bois de feu de la forêt classée de Pahou, SudBénin. Journal de Botanique de la Société Botanique de France. 34 : 81-88.

Biaou H., 1999. Etude des possibilités d'aménagement de la forêt classée de Bassila : Structure et dynamique des principaux groupements végétaux et périodicité d'exploitation. Thèse d'Ingénieur Agronome. Faculté des Sciences agronomiques, UNB. Cotonou, Bénin. 190 pp.

Devineau J.L., 1984. Travaux des chercheurs de la station de LAMTO (Côte-d'Ivoire). Structure et dynamique de quelques forêts tropophiles de l'ouest africain (Côte-d'Ivoire). Programme MAB Savanes. Université d'Abidjan. Station d'Ecologie Tropicale de LAMTO. ISSN 02941422. ISBN 2-7288-0108-8 (5) : 249 pp.

Duplat, P., Perrote, G., 1981. Inventaire et estimation de l'accroissement des peuplements forestiers. Office national des Forêts. Section technique. $432 \mathrm{pp}$.

Dupuy B., Maitre H.-F., N'Guessan A., 1999. Table de production de teck (Tectona grandis). L'exemple de la Côte-d'Ivoire. Bois et Forêts des Tropiques. 261 (3). 7-16.

FAO. 2005. Evaluation des Ressources Forestières Mondiales 2005. FAO. Rome. Italie. 320pp 
Ganglo C.J. \& Lejoly J. \& Pipar T., 1999. Le teck (Tectona grandis L. f.) au Bénin, gestion et perspectives. Bois et Forêts des Tropiques $\mathbf{2 6 1}$ (3): 17-27.

Ganglo C.J., 2005. Groupements de sous-bois, identification et caractérisation des stations forestières: cas d'un bois au Bénin. Bois et Forêts des tropiques 285 (3) : 35-46.

Ganglo J.C. \& de Foucault B., 2006. Plant communities, forest site identification and classification in Toffo reserve, South-Benin. Bois et Forêts des Tropiques. 288 (2) : 25-38.

Gbaguidi F., 1998., Forêts sacrées et conservation de la biodiversité dans le département de l'Ouémé au Sud-Est Bénin. Thèse d'Ingénieur Agronome. FSA/UAC. 161pp.

Gillet F., 2000. Phytosociologie synusiale intégrée. Guide méthodologie. 4 ${ }^{\text {ème }}$ édition revue et corrigée. Université de Neuchâtel - Institut de Botanique. 68 pp.

Gillet F., de Foucault B. \& Julve P., 1991 - La phytosociologie synusiale intégrée: objets et concepts. Candollea 46 : 315-340.

Groulez J., Wood P.-J., 1984. Terminalia superba. Monographie. Centre technique Forestier Tropical. Comonwealth Forestry Institute. 85 pp.

Hessou C., 2003., Contribution à l'aménagement de la forêt classée de l'Ouémé-Boukou: Structure, dynamique des différentes formations et périodicité de coupe. Mémoire de Diplôme d'Etudes Supérieures Spécialisées en Aménagement et Gestion des Ressources Naturelles. Faculté des Sciences Agronomiques, Université d'Abomey-Calavi. $157 \mathrm{pp}+$ annexes.

Hunhyet O.P.K., 2000., Contribution à l'aménagement participatif de la forêt classée des Monts-Kouffé : Structure et dynamique des principaux groupements végétaux. Périodicité de coupe. Thèse d'Ingénieur Agronome. Faculté des Sciences Agronomiques, Université Nationale du Bénin. 131 pp.
Mosango M., 1990. Contribution à l'étude botanique et biogéographique de l'écosystème forêt en région équatoriale (Ile Kongolo, Zaïre). Thèse de Doctorat. Université Libre de Bruxelles. 446 pp.

Noumon, J.C. \& Ganglo J.C., 2005. Phytosociologie appliquée à l'aménagement des forêts : cas du périmètre forestier de Koto (Département du Zou, Centre-Bénin). Acta Botanica Gallica. 152 (3) : 421-426.

Noumon C.J., Ganglo C.J., Azontondé A.H., de Foucault B. \& Adjakidjè V., 2006. Phytocénose à Mallotus oppositifolius (Geisl.) Müll. Arg et Deinbollia pinnata Schumach. \& Thonn. dans le sous-bois des teckeraies du Centre-Bénin. Journal de Botanique de la Société Botanique de France. 36 : 35-61.

Sinadouwirou T., 1997. Forêts sacrées et conservation de la biodiversité : prospection écologique de quelques villages dans le département de l'Atlantique. Thèse d'Ingénieur Agronome FSA/UNB. 160 pp.

Sokpon N., 1995. Recherche écologique sur la forêt dense semi décidue de Pobè au Sud- Est du Bénin : Groupements végétaux, structure, régénération naturelle et chute de litière. Thèse de Doctorat; Université Libre de Bruxelles. 350 pp.

Tohngodo B.C., Ganglo J.C. \& Agbossou K.E., 2006. La phytosociologie comme outil d'identification et de caractérisation des stations forestières. Acta Botanica Gallica. 153 (1) : 135-140.

Tohngodo C., Ganglo C.J., Agbossou K.E., de Foucault B., Adjakidjè V., 2006 Caractéristiques structurales, identification et caractérisation des stations forestières de la forêt classée de Bonou (Sud-est Bénin). Sciences \& Nature. Vol $3 n^{\circ} 1$ : 39-47.

Yayi A.C., 1998. Contribution à l'aménagement de la forêt classée de l'Ouémé supérieur au NordBénin : Structure et dynamique des différents végétaux. Thèse d'Ingénieur Agronome; FSA /UNB ; 144 pp. 